\title{
Switching Energy Dissipation Pathway: in situ Proton-Induced Transformation of AIE-Active Self-assembly to Boost Photodynamic Therapy
}

\author{
Jie $\mathrm{Li}^{\mathrm{a}}{ }^{\mathrm{b}}$, Jianxing Wang ${ }^{\mathrm{a}, \mathrm{b}}$, Jianyu Zhang ${ }^{\mathrm{c}}$, Xiyao $\mathrm{Hu}^{\mathrm{a}}$, Dong Wang, ${ }^{\mathrm{a}, \mathrm{b} *}$, Ben Zhong Tang* \\ ${ }^{a}$ Center for AIE Research, Shenzhen Key Laboratory of Polymer Science and Technology, College of \\ Materials Science and Engineering, Shenzhen University, Shenzhen 518060, China. E-mail: \\ wangd@szu.edu.cn \\ bKey Laboratory of Optoelectronic Devices and Systems of Ministry of Education and Guangdong \\ Province, College of Physics and Optoelectronic Engineering, Shenzhen University, Shenzhen 518060, \\ China. \\ 'Hong Kong Branch of Chinese National Engineering Research Center for Tissue Restoration and \\ Reconstruction, Department of Chemistry, The Hong Kong University of Science and Technology, Clear \\ Water Bay, Kowloon, Hong Kong, China. E-mail: tangbenz@ust.hk
}

\begin{abstract}
With the morphological transformation of fluorescent self-assembly, the functions could be varied simultaneously. However, few attentions have been focused on the function variation in this process. Herein, we presented an aggregation-induced emission (AIE)-active self-assembly to investigate the transformation-induced function variation by switching energy dissipation pathway. This selfassembly was nanosphere with strong emission in neutral condition, indicating that radiative decay dominates the energy dissipation. In acidic circumstance, the sphere transferred to vesicle and nanotube, in which the excited energy was largely consumed by intersystem crossing pathway and afford highly efficient reactive oxygen species (ROS) generation. Particularly, this morphological transformation and functions variation can smoothly proceed in acidic lysosomes, and thus the photodynamic cancer therapy is drastically boosted.
\end{abstract}

Keywords: Aggregation-induced emission (AIE), Self-assembly, Energy conversion, Morphological transformation, Photodynamic therapy (PDT) 
Modulating transformation between different self-assembled nanostructures, e.g., sphere, vesicle, helix and nanotube, is of great significance, because it provides the fundamental understandings of biological self-assembly process, as well as efficient strategies for constructing various supramolecular nanomaterials. ${ }^{[1]}$ Among various kinds of self-assembled systems, of particular interests are fluorescent ones, which have attracted significant scientific interest in the fields of drug delivery, biological or chemical sensor and theranostics. ${ }^{[2]}$ Remarkably, these morphological transformations are often accompanied with quenched fluorescence emission ${ }^{[3]}$ revealing energy consumption pathway transformation from radiative to non-radiative decay. However, this energy dissipation change is usually neglected, nor the applications of the energy dissipated from nonradiative decay. Given that some important functions, such as photodynamic therapy (PDT), are closely related to non-radiative pathways, we are wondering whether the energy dissipated from non-radiative decay involved in morphological transformation could be optimally utilized?

Protonation holds great potential to achieve the variation of energy decay pathways and utilization of non-radiative decay energy ${ }^{[4]}$ The combination of positively charged protons with chromophores is capable of improving the electron donating-accepting (D-A) strength in structure, resulting in the decrease of energy gap ( $\triangle \mathrm{EST})$ between singlet and triplet excited state. Consequently, the non-radiative decay pathway of intersystem crossing (ISC) is established to consume excited-state energy, where reactive oxygen species (ROS) are significantly generated, allowing for PDT. On the other hand, protonation, the strategy of regulating molecular hydrophilicity by changing $\mathrm{pH}$, also presents a feasible protocol for manipulating nanostructures of self-assemblies. ${ }^{[5]}$ More interestingly, as a cellular organelle holding acidic vesicular compartments $(\mathrm{pH} \approx 5)$, lysosome normally receives nano-assemblies via endocytosis, endowing self-assemblies with promising potentials of undergoing in situ morphological transformation driven by protonation in cells. ${ }^{[6]}$ In this regard, switching energy dissipation and promoting non-radiative decay pathway to boost PDT in proton-induced transformation assemblies would be an appealing yet significantly challenging task.

Self-assemblies constructed by traditional luminogens always suffer the inherent obstacle of aggregation-caused fluorescence quenching, which hinder their application as fluorescent materials. On the contrary, aggregation-induced emission (AIE) molecules possess fantastic emission in aggregated states because their twisted structures prevent the strong $\pi-\pi$ stacking, and thus are the 
ideal candidates for fluorescent self-assemblies. ${ }^{[7]}$ Herein, we presented a self-assembly based on AIE amphiphiles that can undergo morphological transformations in acidic conditions to comprehensively study the switch of energy dissipation and variation of functions. The AIE amphiphile TPE-BEP was elaborately designed and synthesized with hydrophilic units of tetraethylene glycol groups and proton-binding sites of pyridine (Scheme S1-S3). As illustrated in Figure 1A, TPE-BEP could self-assemble into nanospheres with strong emission in water due to the typical AIE features (Figure S1). After entering lysosomes of cells, the self-assemblies changed their morphology from nanosphere to vesicle resulting from the protonation of TPE-BEP, concurrently, non-radiative ISC process dominates the energy consumption of excited state. As a result, large amounts of ROS were produced, endowing the self-assemblies with a distinctive performance of killing cancer cells.
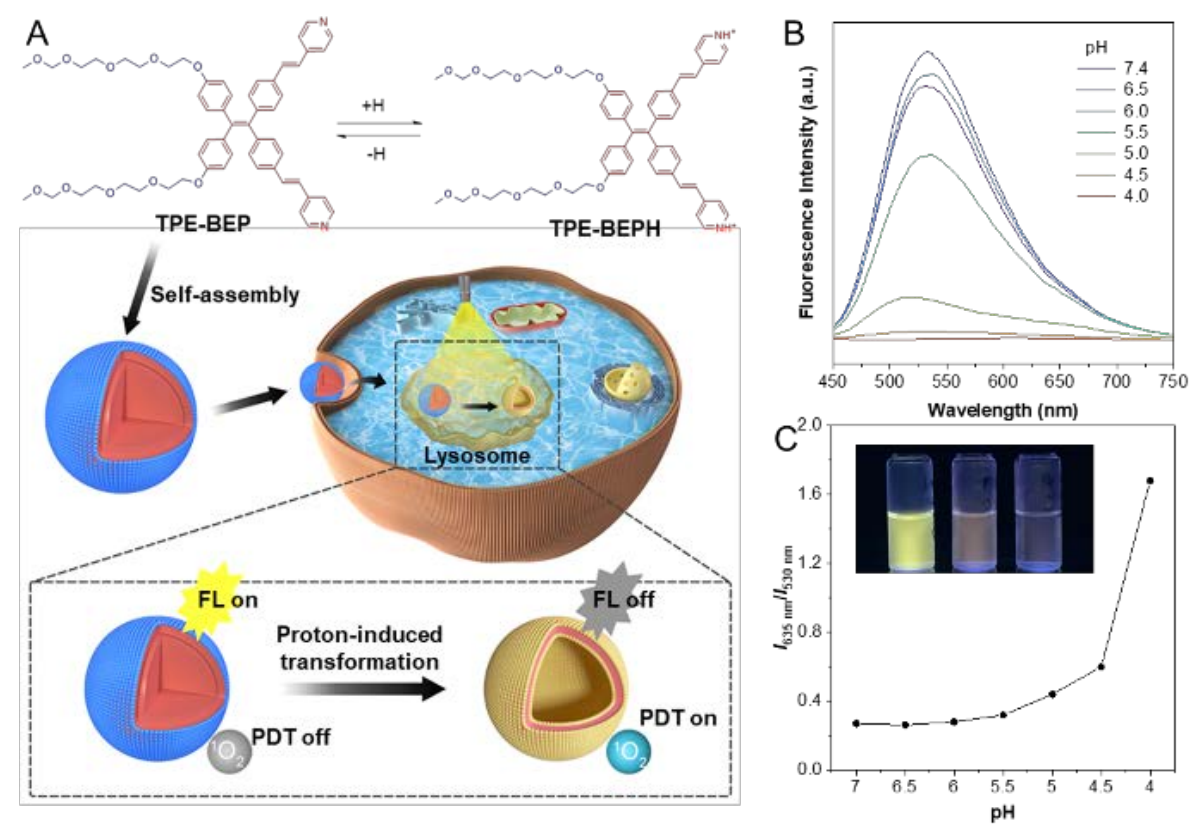

Figure 1. (A) Molecular structures of TPE-BEP and the protonated TPE-BEPH, and the schematic illustration of in situ transformation and energy conversion in cells. (B) Fluorescence spectra of TPE-BEP in PBS with different $\mathrm{pH}$ values. (C) Emission ratios of $635 \mathrm{~nm}$ (TPE-BEPH) and 530 (TPE-BEP) in different pH solutions. [TPE-BEP] = $10 \mu \mathrm{M}$.

Nuclear magnetic resonance (NMR) measurements obviously showed that all the signals of pyridinium ring protons and ethenyl protons of TPE-BEP were downfield shifted after addition of trifluoroacetic acid (Figure S2). This was caused by the decrease of electron density, suggesting the 
protonation. After addition of triethylamine, these signals further reversed back to the initial positions, which indicated the deprotonation. UV-vis and fluorescence spectra also demonstrated the protonation and deprotonation processes (Figure S3). When TPE-BEP was exposed to acidic environment, the UV absorbance changed from 360 to $410 \mathrm{~nm}$ and fluorescence emission simultaneously altered from 530 to $635 \mathrm{~nm}$. On the contrary, the absorption and fluorescence emission were recovered by adding $\mathrm{NaOH}$. This was because combining $\mathrm{H}^{+}$could prompt charge separation and lower energy gap between highest occupied molecular orbital (HOMO) and unoccupied molecular orbital (LUMO), resulting in red shifts of both absorbance and emission. Density functional theory (DFT) calculation revealed that HOMO and LUMO electron distribution of TPE-BEPH (protonated TPE-BEP) were separated to a much larger extent than TPE-BEP, and the corresponding energy gap between HOMO and LUMO of TPE-BEPH was $4.39 \mathrm{eV}$, which was lower than $5.14 \mathrm{eV}$ of TPE-BEP (Figure S4). This solidly confirmed the protonation of TPE-BEP and the long emission wavelength $(635 \mathrm{~nm})$ of TPE-BEPH.

To gain a deep insight into the protonation process, fluorescence emission of TPE-BEP in aqueous solutions with different $\mathrm{pH}$ values was systematically investigated. The pKa of TPE-BEP was firstly measured to be 5.6, according to the results of UV absorbance via pH values (Figure S5). As depicted in Figure 1B and 1C, TPE-BEP solution showed yellow emission of $530 \mathrm{~nm}$ at $\mathrm{pH}=$ 7.4, where TPE-BEP was seldom protonated with an extremely low $\mathrm{I}_{635} \mathrm{~nm} / \mathrm{I}_{530} \mathrm{~nm}$ ratio. However, upon gradually decreasing the $\mathrm{pH}$ values, the emission intensity at $530 \mathrm{~nm}$ stepwise reduced and the ratios of $\mathrm{I}_{635 \mathrm{~nm}} / \mathrm{I}_{530 \mathrm{~nm}}$ obviously enhanced owing to the protonation of TPE-BEP. Species distributions of TPE-BEP and TPE-BEPH in Figure S6 displayed the progressive decrease of TPEBEP and growth of TPE-BEPH over $\mathrm{pH}$ reduction. For example, 72\% of TPE-BEP existed with the unprotonated form while $28 \%$ was protonated TPE-BEPH at $\mathrm{pH}=6$, where the ratios of $1635 \mathrm{~mm} / \mathrm{I530}$ $\mathrm{nm}$ increased to 0.28 . Consequently, the solution emission turned to orange at $\mathrm{pH}=5$, and it further became red with an emission peak of $635 \mathrm{~nm}$ when $\mathrm{pH}$ was 4 owing to the complete protonation.

In virtue of amphiphilic molecular structure, TPE-BEP could spontaneously form nanoaggregates in water, and the self-assembly behaviors in various $\mathrm{pH}$ were evaluated. Transmission electron microscopy (TEM) and dynamic laser scattering (DLS) measurements showed that the aggregates of TPE-BEP in $\mathrm{pH}=7.4$ solution were well-defined spheres with a diameter of $220 \mathrm{~nm}$ (Figure 2A and 2D). CLSM further demonstrated that the spherical self-assemblies emitted yellow 
fluorescence (Figure S7). Atomic force microscopy (AFM) results confirmed the spherical structure, and the height profile demonstrated that the collapsed spheres may possess a multilamellar configuration (Figure S8) ${ }^{[8]}$ Moreover, upon adding $\mathrm{H}_{2} \mathrm{O}$ to good solvent DMF of TPE-BEP (Figure S9), pronounced red shifts of both absorbance and emission were observed, which suggested a possible J-aggregated stacking in spherical structure. Similarly, at pH = 5 condition, the morphology of TPE-BEP assemblies were vesicles with a diameter of $460 \mathrm{~nm}$ (Figure 2B). AFM images also displayed the hollow vesicular structure, as well as the multilamellar structure. When the $\mathrm{pH}$ value was 4, red-emissive nanotubes with $100 \mathrm{~nm}$ of length and $5 \mathrm{~nm}$ of width were obtained (Figure 2C).
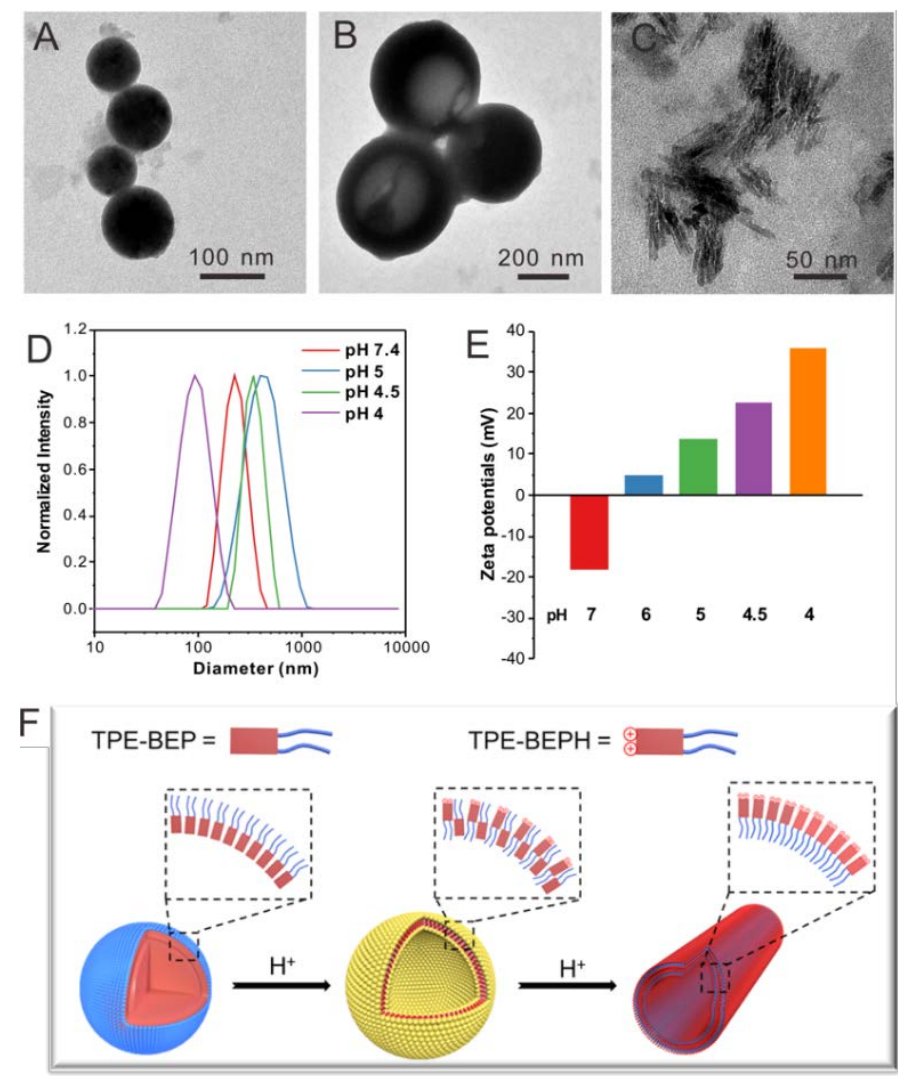

Figure 2. TEM images of TPE-BEP self-assemblies in various pH conditions: (A) 7.4, (B) 5.0 and (C) 4.0. (D) DLS results and (E) Zeta potentials of TPE-BEP self-assemblies in various $\mathrm{pH}$ conditions. (F) Schematic illustration of the transformation between TPE-BEP self-assemblies driven by protons.

The mechanism of morphological transformations was further explored (Figure 2F). It was observed that the Zeta potential of TPE-BEP self-assemblies (Figure 2E) at $\mathrm{pH}=7.4$ was determined to be $-18 \mathrm{mV}$, implying that tetraethylene glycol groups located on the outer shell of nanospheres because hydrophilic tetraethylene glycol group normally displayed negative charges in water. 
Nevertheless, the potentials of TPE-BEP self-assemblies changed from negative to positive when gradually deceasing the $\mathrm{pH}$ values, and the positive potentials even raised over the fall of $\mathrm{pH}$. Seeing that TPE-BEP was protonated in acidic conditions and converted to the positively charged TPE$\mathrm{BEPH}$, it was reasonable to infer that the protonated pyridinium of TPE-BEPH positioned on the outer layer of assemblies instead of tetraethylene glycol group, neutralizing the negative charges and further triggering the charge reversal. For example, when $\mathrm{pH}$ was 5, 80\% of TPE-BEP was converted to the positively charged TPE-BEPH, and positively charged pyridinium units dominated the outer shell of vesicles, resulting in a rather positive zeta potential. The perfect overlap between yellow and red colors in CLSM images of $\mathrm{pH}=5$ proved this coexistence of TPE-BEP and TPE$\mathrm{BEPH}$ in one self-assembly (Figure S7). Additionally, the Zeta potential value of $\mathrm{pH}=5$ was much less than $\mathrm{pH}=7.4$, indicating a weaker repulsive interaction at $\mathrm{pH}=5$, and the nanosphere sphere trended to swell and gave birth to hollow vesicles. Furthermore, when all of TPE-BEP were converted to TPE-BEPH ( $\mathrm{pH}=4)$, the outer layer of self-assemblies was all pyridinium units and thus exhibited extremely highly positive Zeta potentials, resulting in strong electrostatic repulsion and subsequent formation of nanotubes. The rapid decrease of lifetime of TPE-BEP assemblies from $\mathrm{pH}=7.4$ (2.14 ns) to $\mathrm{pH}=4.0$ (0.07 ns) verified the loosely stacking state and electrostatic repulsion of TPE-BEP assemblies in acidic conditions (Figure S10).

In the morphological transformations, the fluorescence intensity and quantum yield (QY) of TPE-BEP remarkably reduced from sphere (pH 7.4, 13.2\%) to vesicle (pH 5.0, 8.8\%) to nanotubes (pH 4.0, 1.2\%) (Figure 3A). This suggested a critical conversion of dissipating excitation energy during transformation.

The energy dissipation process was further investigated. Theoretical calculation of the energy gap between singlet and triplet states was conducted. ${ }^{[9]}$ As depicted in Figure 3B, protonated TPEBEPH possessed a rather low $\triangle$ EST $(0.11 \mathrm{eV})$ than TPE-BEP $(0.55 \mathrm{eV})$, which meant that TPEBEPH could open up a pathway of ISC process to dissipate the excitation energy, suggesting the potential of efficient ROS production. 9,10-Anthracenediyl-bis(methylene)dimalonic acid (ABDA) was used as the ROS indicator. As shown in Figure 3C and S11, scarcely any of ROS was detected at $\mathrm{pH}=7.4$ in the presence of both TPE-BEP and light irradiation. Upon in acidic solutions, ROS was indeed produced and a lower $\mathrm{pH}$ condition induced a higher ROS generation efficiency on account that more TPE-BEP was protonated to TPE-BEPH. The performance of ROS generation in 
$\mathrm{pH}=4$ was even better than that of Rose Bengal, reflecting a promising potential for PDT application. Furthermore, TPE-BEPMe was synthesized as a control to modulate the feature of protonated TPE-BEPH (Scheme S4). UV-vis and fluorescence spectra exhibited that TPE-BEPMe and TPE-BEPH had the approximated both absorption and emission (Figure S12). In addition, theoretical calculation demonstrated that they shared the same energy gap between HOMO and LUMO, as well as $\triangle \mathrm{EST}$ (Figure S13-14). Moreover, TPE-BEPMe showed the similar ROS generation efficiency with TPE-BEPH, but much higher than TPE-BEP (Figure S15). These results solidly confirmed that protonated TPE-BEPH possessed efficient ISC pathway to consume excitedstate energy with an excellent capacity of generating ROS while TPE-BEP did not.

The energy dissipation conversion accompanying morphological transformation can be illustrated as Figure 3D. TPE-BEP self-assembled into spheres and the luminophores tensely packed in the core, leading to the excited-state energy dissipated through radiative decay pathway with strong emission. When exposed to acidic circumstance, due to the protonation of TPE-BEP, the selfassembles transferred into nanotubes where the luminophores placed in the outer shell, and the corresponding $\triangle \mathrm{EST}$ of TPE-BEP decreased, opening ISC pathway to consume excited energy. In brief, the radiative decay pathway converted to ISC during morphological transformation driven by protonation
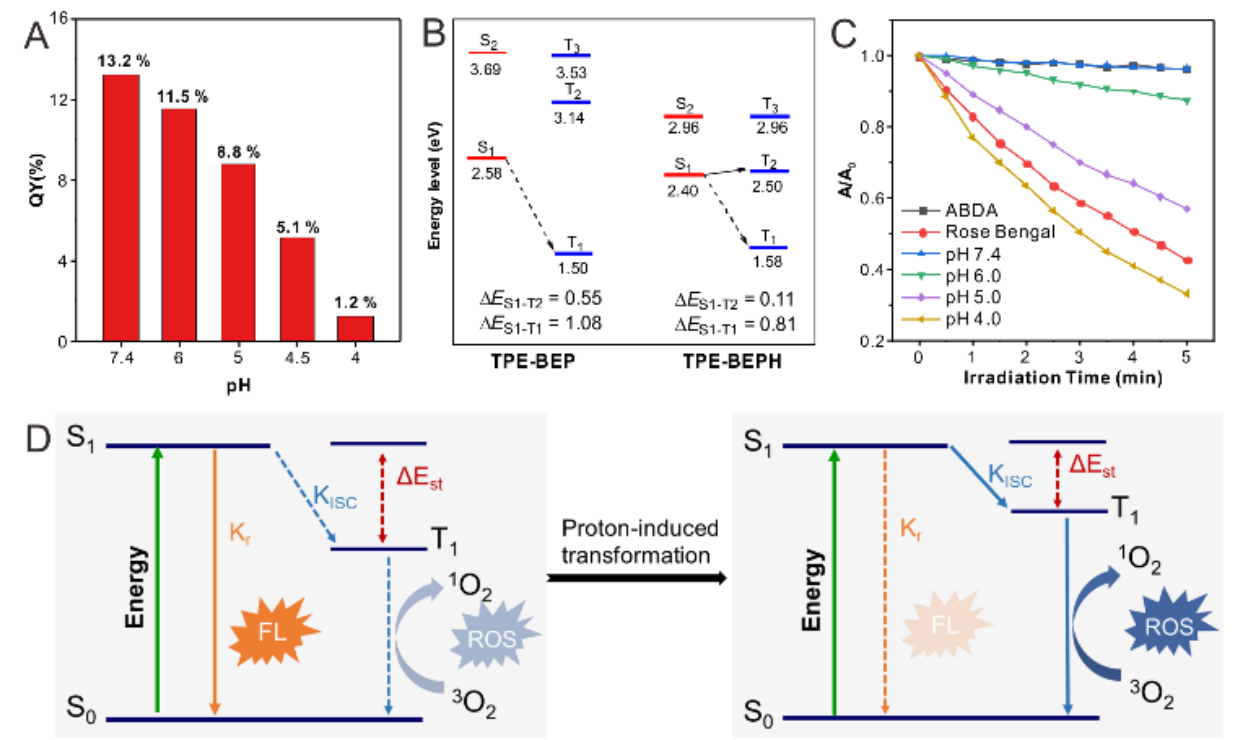

Figure 3 (A) Quantum yield (QY) of TPE-BEP in water solutions with different pH. (B) Calculated energy diagrams of TPE-BEP and TPE-BEPH. (C) ROS generation of TPE-BEP in different $\mathrm{pH}$ solution indicated by ABDA. (D) Jablonski diagram of radiative and ISC processes before and after 
proton-induced transformation of TPE-BEP self-assemblies. $\mathrm{S}_{0}$ : the ground state, $\mathrm{S}_{1}$ : the lowest excited singlet state, $\mathrm{T}_{1}$ : the lowest excited triplet state. $\mathrm{K}_{\mathrm{r}}$, and $\mathrm{K}_{\mathrm{ISC}}$ are the rate constants of the radiative decay and the ISC process, respectively. FL: fluorescence. [TPE-BEP] $=10 \mu \mathrm{M}$.

Next, cell imaging was proposed to check the proton-induced transformation of TPE-BEP in cells. As demonstrated in Figure 4A and S16, after treating 4T1 cancer cells with TPE-BEP selfassemblies, bright yellow emission was observed in cells, and the fluorescence signal remarkably enhanced when prolonging the incubation time to 2 hours. This indicated that TPE-BEP selfassemblies were efficiently taken up by cells. However, no red signal, referring to TPE-BEPH, could be detected when incubated for 0.5 hour. The red emission appeared at 1 hour and its intensity further increased over time, in which the yellow emission intensity decreased simultaneously, which reflected that spherical TPE-BEP assemblies might transfer to vesicles. Co-location images further showed that both yellow and red assemblies were mainly distributed in lysosome (Figure 4B and S17-18), suggesting that TPE-BEP self-assemblies were taken up through endocytosis and the transformation happened in lysosomes.

To observe the in situ transformation in lysosomes, intracellular $\mathrm{pH}$ values were regulated by various PH PBS containing nigericin (Figure S19). TPE-BEP assemblies were incubated for 2 hours with following treatment with different $\mathrm{pH}$ PBS. The yellow fluorescence emission in cells decreased dramatically with reduction of $\mathrm{pH}$ values, while the red emission remarkably enhanced. This $\mathrm{pH}$-dependent alteration verified the transformations induced by protonation in cells. Additionally, aiming to obtain real-time transformation in cells, acetic acid that can permeate the cell membrane was selected to prompt the protonation of TPE-BEP. ${ }^{[10]}$ As shown in Figure S20, the red areas greatly expanded and the red signal became much stronger over time, accompanied with decay of yellow fluorescence, which clearly indicated the in situ transformation in cells. 

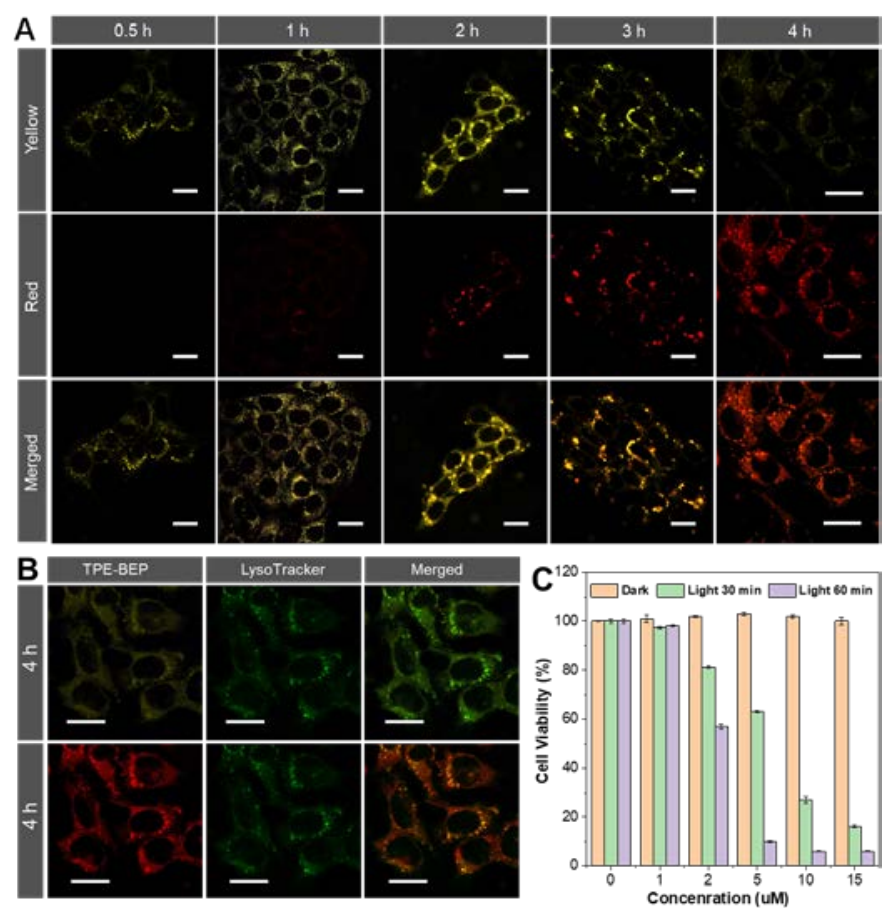

Figure 4. (A) Cell images of 4T1 cells after incubation with TPE-BEP self-assemblies for various periods. (B) Co-location of 4T1 cells stained with LysoTracker after incubation with TPE-BEP selfassemblies. $\lambda_{\text {ex }}: 405 \mathrm{~nm}$; $\lambda_{\text {em }}$ (yellow): 500-550 nm and $\lambda_{\text {em }}$ (yellow): 600-650 nm. [TPE-BEP] $=10$ $\mu \mathrm{M}$. Scale bar is $10 \mu \mathrm{m}$. (C) $4 \mathrm{~T} 1$ cell viability after incubation with different concentrations of TPEBEP self-assemblies in the dark or under irradiation.

Finally, the application of ROS generated by ISC pathway was tested in cancer cells. Compared to negligible emission of ROS indicator dichlorofluorescein (DCFH-DA) or TPE-BEP assemblies alone, bright fluorescence was found in cells when treated with both DCFH-DA and TPE-BEP assemblies after irradiation (Figure S21), reflecting an efficient ROS generation in cells. Moreover, when adjusting the intracellular $\mathrm{pH}$ to 7.4 , the cells showed considerably weak emission after irradiation. Inversely, the emission at intracellular $\mathrm{pH}=4$ was evidently stronger than that of the cell without adjusting, which suggested that the ROS generation was triggered by protonation. The produced ROS could cause apoptosis of cancer cells (Figure S22). Dose-dependent cytotoxicity showed that cancer cell viability was gradually and rapidly decreased upon raising the TPE-BEP concentration, and the longer irradiation time caused a much lower viability (Figure 4C and S23). The effective PDT protocol was successfully extended to other cancer cells, such as HeLa cells and HepG2 cells (Figure S24). Together with the excellent photostability (Figure S25), TPE-BEP 
assemblies present excellent nano-photosensitizers for PDT application.

In summary, we have developed an AIE-active self-assembly that exhibited proton-induced morphological transformation to study the energy dissipation conversion and function variation. The self-assembly had spherical structure in neutral condition and dissipated excited-state energy via radiative pathway with strong emission. Due to protonation in acidic circumstance, the nanospheres transferred to nanovesicles and nanotubes but with quite weak emission, where chromophore located in incompact outershell. Concurrently, the protonation reduced the energy gap $\triangle \mathrm{EST}$, opening up ISC pathway of producing ROS to consume the excited-state energy. The morphological transformation and energy dissipation pathway conversion can be achieved in acidic lysosome, and the high-performance ROS production enable the self-assembly to be drastically efficient for ablating cancer cells by means of PDT. This study would thus provide an innovative insight into relationship between functions, energy dissipation and morphologies of self-assembly systems, and facilitate the practical applications of fluorescent self-assemblies.

\section{Supporting information}

Supporting information for this article is available on the website.

\section{Conflict of interest}

The authors declare no conflict of interest.

\section{Acknowledgements}

This work was financially supported by China Postdoctoral Science Foundation (2019M653005), the National Natural Science Foundation of China (21801169, 21902106), and the Developmental Fund for Science and Technology of Shenzhen government (JCYJ20190808153415062), the Natural Science Foundation for Distinguished Young Scholars of Guangdong Province (2020B1515020011). The authors also acknowledge the Instrumental Analysis Center of Shenzhen University.

\section{Reference}

[1] a) W. Zhang, T. Aida, Science 2012, 337, 1462-1463; b) R. Freeman, M. Han, Z. Álvarez, J. A. Lewis, J. R. Wester, N. Stephanopoulos, M. T. McClendon, C. Lynsky, J. M. Godbe, H. Sangji, 
E. Luijten, S. I. Stupp, Science 2018, 362, 808-813; c) D. Mozhdehi, K. M. Luginbuhl, J. R. Simon, M. Dzuricky, R. Berger, H. S. Varol, F. C. Huang, K. L. Buehne, N. R. Mayne, I. Weitzhandler, M. Bonn, S. H. Parekh, A. Chilkoti, Nat. Chem. 2018, 10, 496-505; d)C. Yuan, W. Ji, R. Xing, J. Li, E. Gazit, X. Yan, Nat. Rev. Chem. 2019, 3, 567-588; e) S. Chen, F. K.-C. Leung, M. C. A. Stuart, C. Wang, B. L. Feringa, J. Am. Chem. Soc. 2020, 142, 10163-10172; f) T. Fukui, S. Kawai, S. Fujinuma, Y. Matsushita, T. Yasuda, T. Sakurai, S. Seki, M. Takeuchi, K. Sugiyasu, Nat. Chem. 2017, 9, 493-499; g) M. Al Kobaisi, S. V. Bhosale, K. Latham, A. M. Raynor, S. V. Bhosale, Chem. Rev. 2016, 116, 11685-11796.

[2] a) J. Li, J. Wang, H. Li, N. Song, D. Wang, B. Z. Tang, Chem. Soc. Rev. 2020, 49, 1144-1172; b) W. Guan, W. Zhou, C. Lu, B. Z. Tang, Angew. Chem. Int. Ed. 2015, 54, 15160-15164; c) Z. Wang, J. Nie, W. Qin, Q. Hu, B. Z. Tang, Nat. Commun. 2016, 7, 12033.

[3] a) R. Dong, B. Zhu, Y. Zhou, D. Yan, X. Zhu, Angew. Chem. Int. Ed. 2012, 51, 11633-11637; b) J. Wang, J. Li, Y. Li, Z. Zhang, L. Wang, D. Wang, L. Su, X. Zhang, B. Z. Tang, Chem. Sci. 2020, 11, 6472-6478; c) J. Li, K. Liu, Y. Han, B. Z. Tang, J. Huang, Y. Yan, ACS Appl. Mater. Interfaces 2016, 8, 27987-27995.

[4] a) Q. Tang, W. Xiao, C. Huang, W. Si, J. Shao, W. Huang, P. Chen, Q. Zhang, X. Dong, Chem. Mater. 2017, 29, 5216-5224; b) J. Tian, L. Ding, H.-J. Xu, Z. Shen, H. Ju, L. Jia, L. Bao, J.-S. Yu, J. Am. Chem. Soc. 2013, 135, 18850-18858; c) J. F. Lovell, T. W. B. Liu, J. Chen, G. Zheng, Chem. Rev. 2010, 110, 2839-2857.

[5] a) M. T. Fenske, W. Meyer-Zaika, H.-G. Korth, H. Vieker, A. Turchanin, C. Schmuck, J. Am. Chem. Soc. 2013, 135, 8342-8349; b) H. Dou, M. Jiang, H. Peng, D. Chen, Y. Hong, Angew. Chem. Int. Ed. 2003, 42, 1516-1519.

[6] a) J. Zhan, Y. Cai, S. He, L. Wang, Z. Yang, Angew. Chem. Int. Ed. 2018, 57, 1813-1816; b) Y. Cong, L. Ji, Y.-J. Gao, F.-H. Liu, D.-B. Cheng, Z. Hu, Z.-Y. Qiao, H. Wang, Angew. Chem. Int. Ed. 2019, 58, 4632-4637; c) M. Borkowska, M. Siek, D. V. Kolygina, Y. I. Sobolev, S. Lach, S. Kumar, Y.-K. Cho, K. Kandere-Grzybowska, B. A. Grzybowski, Nat. Nanotechnol. 2020, 15, 331-341; d) X. Li, C. y. Kim, S. Lee, D. Lee, H.-M. Chung, G. Kim, S.-H. Heo, C. Kim, K.-S. Hong, J. Yoon, J. Am. Chem. Soc. 2017, 139, 10880-10886.

[7] a) H. Zhang, X. Zheng, R. T. K. Kwok, J. Wang, N. L. C. Leung, L. Shi, J. Z. Sun, Z. Tang, J. W. Y. Lam, A. Qin, B. Z. Tang, Nat. Commun. 2018, 9, 4961; b) H. Zhang, Z. Zhao, A. T. Turley, L. Wang, P. R. McGonigal, Y. Tu, Y. Li, Z. Wang, R. T. K. Kwok, J. W. Y. Lam, B. Z. Tang, Adv. Mater. 2020, 32, 2001457; c) D. Wang, B. Z. Tang, Acc. Chem. Res. 2019, 52, 2559-2570; d) B. Z. Tang, X. Zhan, G. Yu, P. P. Sze Lee, Y. Liu, D. Zhu, J. Mater. Chem. 2001, 11, 2974-2978.

[8] B.-P. Jiang, D.-S. Guo, Y.-C. Liu, K.-P. Wang, Y. Liu, ACS Nano 2014, 8, 1609-1618.

[9] a) G. Feng, G.-Q. Zhang, D. Ding, Chem. Soc. Rev. 2020, DOI: 10.1039/D0CS00671H; b) C. Chen, X. Ni, H.-W. Tian, Q. Liu, D.-S. Guo, D. Ding, Angew. Chem. Int. Ed. 2020, 59, 1000810012; c) S. Liu, H. Zhang, Y. Li, J. Liu, L. Du, M. Chen, R. T. K. Kwok, J. W. Y. Lam, D. L. Phillips, B. Z. Tang, Angew. Chem. Int. Ed. 2018, 57, 15189-15193; d) J.-S. Ni, T. Min, Y. Li, M. Zha, P. Zhang, C. L. Ho, K. Li, Angew. Chem. Int. Ed. 2020, 59, 10179-10185.

[10] a) S. Liu, X. Zhou, H. Zhang, H. Ou, J. W. Y. Lam, Y. Liu, L. Shi, D. Ding, B. Z. Tang, J. Am. Chem. Soc. 2019, 141, 5359-5368; b) S. Gao, G. Wei, S. Zhang, B. Zheng, J. Xu, G. Chen, M. Li, S. Song, W. Fu, Z. Xiao, W. Lu, Nat. Commun. 2019, 10, 2206. 